anales de psicología, 2012, vol. 28, $\mathrm{n}^{\circ} 2$ (mayo), 567-575 http://dx.doi.org/10.6018/analesps.28.2.124111
C) Copyright 2012: Servicio de Publicaciones de la Universidad de Murcia. Murcia (España) ISSN edición impresa: 0212-9728. ISSN edición web (http://revistas.um.es/analesps): 1695-2294

\title{
Inteligencia emocional y género: más allá de las diferencias sexuales
}

\author{
Leire Gartzia ${ }^{1 *}$, Aitor Aritzeta ${ }^{2}$ Nekane Balluerka ${ }^{2}$ E Esther Barberá ${ }^{3}$ \\ ${ }^{1}$ Universidad de Deusto (España) \\ ${ }^{2}$ Universidad del País V asco (España) \\ ${ }^{3}$ Universidad de Valencia (España)
}

\begin{abstract}
Resumen: La mayoría de los estudios sobre género e Inteligencia Emocional (IE) se han centrado en analizar diferencias en función del sexo y han mostrado resultados contradictorios. Con objeto de formular nuevas propuestas de análisis en este ámbito de estudio, el presente trabajo examina el efecto de la identidad de género sobre la IE en una muestra de 338 trabajadores/as. Para la medida de la IE se utilizaron tanto medidas de auto-percepción (TMMS) como de habilidad (MSCEIT). Los resultados indican que la aceptación de rasgos de expresividad por parte de las mujeres podría explicar sus mayores puntuaciones en IE. Además, los resultados muestran que las personas andróginas, en comparación con las instrumentales y expresivas, presentan niveles superiores de IE. A partir de tales resultados, se pone de manifiesto la necesidad de ir más allá del enfoque de las diferencias sexuales en IE y de promover referentes de identidad de género menos estereotipados.
\end{abstract}

Palabras clave: identidad de género; inteligencia emocional; androginia.

Title: Emotional intelligence and gender: beyond sex differences.

Abstract: Research on gender and Emotional Intelligence (EI) has analysed individual differences in relation to sex and has yielded contradictory results. With the aim of suggesting new proposals in this field of study, the present work analyses the influence of gender identity on EI in a sample of 338 workers. We combined self-report measures (TMMS) and ability based measures (MSCEIT) of Emotional Intelligence. Results indicate that women's higher acceptance of expressive traits may help to explain their higher scores on EI. Also, results show that androgynous individuals, compared with instrumental and expressive individuals, present higher levels of EI. Taking into account these results, the need to go beyond the "sex differences" approach when analysing EI and to develop less stereotyped gendered identity references is discussed.

Key words: gender identity; emotional intelligence; androgyny.

\section{Introducción}

La Inteligencia Emocional (IE) (Salovey y Mayer, 1990) es un constructo de gran interés en la investigación psicosocial que incluye competencias emocionales relacionadas con la capacidad para atender a los sentimientos y comprenderlos con claridad, así como para regular los estados emocionales negativos y prolongar los positivos (Salguero, FernándezBerrocal, Ruiz-Aranda y Cabello, 2009). Aunque este tipo de inteligencia incluye en su definición competencias emocionales directamente relacionadas con los roles de género (Sánchez, Fernández-Berrocal, Montañés y Latorre, 2008), no está clara la relación existente entre el género y la IE, siendo necesario profundizar en dicha línea de investigación (Petrides, Furnham, y Martin, 2004; Salovey, 2006).

Si bien son muchos los trabajos llevados a cabo con objeto de analizar las diferencias en función del sexo desde el estudio de la IE, los resultados observados han sido contradictorios (Brackett y Salovey, 2006). En términos generales, se ha concluido que las mujeres poseen mayores competencias de IE (Joseph y Newman, 2010). Estas diferencias son especialmente marcadas en el caso de la IE evaluada a partir de modelos que analizan la IE como un conjunto de habilidades cognitivas (Mayer, Salovey y Caruso, 2000). Desde estos modelos, se encuentran diferencias entre hombres y mujeres en el uso, comprensión y manejo de las emociones, obteniendo las mujeres puntuaciones mayores (Mayer, Salovey y Caruso, 2000; Salovey y Mayer, 1990; Salovey, 2006). Desde los modelos que operacionalizan la IE como un conjunto de competencias emocionales analizadas mediante medidas autopercibidas, no se encuentran diferencias tan claras en los niveles generales de IE, aunque sí en algunas de sus subdi-

Dirección para correspondencia [Correspondence address]: Leire Gartzia. Dirección postal: c/ Avenida de las Universidades, 24, 48007 Bilbao (Bizkaia, España). E-mail: leire.gartzia@deusto.es mensiones. Por ejemplo, las mujeres tienden a mostrar puntuaciones superiores en las ramas de atención y expresión emocional y los hombres en las ramas de regulación (BarOn, 2006).

De acuerdo con lo que se acaba de señalar, numerosas investigaciones apuntan que es necesario ahondar en la línea de investigación que aborda el estudio del género y la IE para determinar cuáles son las causas de las diferencias observadas entre los hombres y las mujeres (Candelá, Barberá, Ramos y Sarrió, 2002; Conway, 2000; Dawda y Hart, 2000; Joseph y Newman, 2010; Petrides, Furnham, y Martin, 2004; Salovey, 2006). Concretamente, algunos estudios han propuesto analizar variables relacionadas con patrones de socialización y desempeño de distintos roles (Brackett y Salovey, 2006). Teniendo en cuenta que la identidad de género ${ }^{1}$ - entendida como la identificación con rasgos estereotípicamente masculinos (instrumentales) y rasgos estereotípicamente femeninos (expresivos) (Parsons y Bales, 1955)-, está directamente relacionada con la socialización diferencial de mujeres y hombres y es una de las variables que determinan en mayor medida la forma de pensar, comportarse y sentir (Bem, 1974; Tajfel y Turner, 1986), ésta podría ayudar a explicar las diferencias sexuales en IE.

La expresividad incluye rasgos estereotípicamente femeninos de personalidad directamente relacionados con la IE, tales como la sociabilidad, la atención a las necesidades de los demás, la sensibilidad o la empatía. Por su parte, la instrumentalidad incluye rasgos estereotípicamente masculinos como la independencia, la asertividad, la alta orientación a la

${ }^{1}$ El término identidad de género puede adoptar distintos significados. Dado que el género se define como un sistema social que incluye roles y estereotipos de género y que influye en la definición de la identidad en relación a funciones expresivas e instrumentales (Stewart y McDermott, 2004; Bem, 1974; Spence y Helmreich, 1978), en este trabajo nos referiremos a la identidad de género como la parte del autoconcepto que incluye tales rasgos (expresivos e instrumentales). 
tarea o la ambición. Diversos estudios han mostrado que la identificación con dichos rasgos de género es un factor relevante a la hora de explicar diferencias sexuales en distintas variables psicológicas y culturales (Dambrun, Duarte y Guimond, 2004; Hyde, 2005; Randel, 2002; Bourne y Maxwell, 2010), así como en la experiencia y expresión emocional (Fischer, 1993).

Asumir que los rasgos de identidad de género constituyen una potencial variable explicativa de las diferencias entre mujeres y hombres en IE implica asumir, en primer lugar, que existen diferencias sexuales en dichos rasgos de identidad. En otras palabras, supone asumir que las mujeres se identifican con rasgos de expresividad en mayor medida que los hombres, mientras que los hombres se identifican con rasgos de instrumentalidad en mayor medida que las mujeres (Bem, 1974). Sin embargo, existen ciertas contradicciones en los estudios que analizan dichas diferencias. Así, en algunos estudios se ha sugerido que, en la actualidad, no existen diferencias entre mujeres y hombres en la aceptación de rasgos de expresividad e instrumentalidad, planteándose que la adopción de rasgos expresivos e instrumentales depende de los roles sociales, laborales y/o profesionales que se ocupan en la sociedad, y no del sexo (Echebarria, 2010). Otros estudios, por el contrario, han sugerido que, aunque no existen diferencias sexuales en instrumentalidad, la aceptación de rasgos de expresividad sigue siendo mayor por parte de las mujeres que por parte de los hombres (Twenge, 1997; 2001).

Desde estos estudios, se ha sugerido que los cambios en la función social de la mujer en nuestra sociedad, que han favorecido su presencia en el mercado laboral y en contextos públicos tradicionalmente ocupados por los hombres, han dado lugar a que las diferencias en instrumentalidad tiendan a desaparecer. Sin embargo, no sucede lo mismo en el caso de la expresividad. Así, dada la menor incorporación de los hombres a funciones tradicionalmente femeninas en el ámbito privado y dado que los cambios experimentados por los hombres han sido menores a los experimentados por las mujeres (Twenge, 1997; 2001), las puntuaciones de los hombres en expresividad tienden a seguir siendo inferiores. Estos resultados son coherentes con la teoría del rol social y la congruencia de rol propuesta por Eagly (Eagly, 1987, Eagly y Karau, 2002), según la cual los roles sexuales establecidos en la sociedad de manera rígida obstaculizan el desarrollo de rasgos de identidad contra-estereotípicos. En base a estos estudios, esperamos encontrar diferencias sexuales en la aceptación de rasgos de expresividad y, como veremos en el siguiente apartado, son dichas diferencias las que podrían explicar las diferencias entre mujeres y hombres en IE.

\section{Hipótesis 1: Las mujeres mostrarán mayores puntua- ciones que los hombres en los rasgos de identidad aso- ciados a la IE (rasgos expresivos)}

\section{Elpapel mediador de la expresividad}

Como hemos apuntado, las diferencias sexuales en IE podrían deberse a que las mujeres se identifican, en mayor medida que los hombres, con rasgos de identidad expresivos. Para establecer esta predicción, partimos de la base de que la socialización en lo femenino implica el desarrollo de rasgos de identidad expresivos estrechamente asociados a un gran número de competencias de IE, tales como la sensibilidad, la sociabilidad o la atención a las necesidades de los demás (Stewart y McDermont, 2004). De hecho, los rasgos de expresividad coinciden con el desarrollo de características emocionales de orientación interpersonal (Conway, 2000), la disposición hacia el cuidado y expresión emocional (Wester, Vogel, Pressly y Heesacker, 2002) y la empatía (Hoffman, 1977). Además, se ha encontrado que las personas que puntúan alto en dichos rasgos estereotípicamente femeninos de expresividad (es decir, que tienen una identidad de género más expresiva) tienden a procesar emociones de manera más efectiva (Bourne y Maxwell, 2010) y a buscar y obtener más apoyo emocional (Reevy y Maslach, 2001). Estos resultados sugieren que, independientemente del sexo, la identificación con rasgos de identidad estereotípicamente femeninos (expresivos) se asocia con el desarrollo de competencias emocionales de orientación interpersonal (Fischer, 1993). En línea con esta idea, se ha demostrado que la comunicación de emociones complejas también resulta más efectiva entre las personas con identidad de género expresiva o estereotípicamente femenina (Friedman y Riggio, 1999). De igual for$\mathrm{ma}$, se ha observado que el rechazo de las dimensiones emocionales consideradas como femeninas y relacionadas con la expresividad, supone un obstáculo para una vivencia completa de la experiencia emocional por parte de los hombres (Blazina, 2001).

Teniendo en cuenta que la mayor parte de los estudios que examinan variables relacionadas con el género lo hacen incluyendo únicamente la variable sexo (Hyde, 2005; Stewart y McDermott, 2004), el análisis del papel mediador de los rasgos de expresividad a la hora de explicar las mayores puntuaciones en IE por parte de las mujeres puede aportar resultados esclarecedores en este sentido. Por ello, partiendo de la base de que rasgos estereotípicamente femeninos (tales como la sociabilidad, la empatía o la capacidad de atender a las necesidades de otras personas) se relacionan con muchas de las competencias emocionales incluidas en la definición de la IE, se plantea como hipótesis que una de las causas de que las mujeres presenten mayores puntuaciones en IE que los hombres podría ser su mayor aceptación de rasgos de identidad estereotípicamente femeninos (expresivos).

Hipótesis 2: La expresividad mediará la relación entre el sexo y la IE.

\section{Androginia e Inteligencia Emocional}

Hasta ahora, hemos destacado el papel de la expresividad en el desarrollo de competencias de IE y hemos argumentado que dichos rasgos podrían explicar las mayores puntuaciones de las mujeres en IE. Para ello, hemos apuntado que los rasgos estereotípicamente femeninos, previsiblemente presentes en mayor medida entre las mujeres, favorecen im- 
portantes competencias emocionales (Conway, 2000; Hoffman, 1977; Wester et al., 2002). Sin embargo, esta perspectiva no implica afirmar que los rasgos de instrumentalidad no tengan valor en el desarrollo de la IE. En este sentido, algunos estudios han mostrado que las personas que integran en su identidad rasgos de instrumentalidad tales como la asertividad, la independencia o la autoconfianza son, independientemente de su sexo, menos propensas que las personas expresivas a centrarse en emociones negativas (Conway, Giannopoulos, y Stiefenhofer, 1990). Además, los individuos instrumentales presentan mayores niveles de autoestima (Helgeson, 1994). Esta perspectiva ayuda a comprender por qué los hombres son habitualmente mejores a la hora de regular algunas emociones negativas como la culpa o la ansiedad (Brody y Hall, 2000) y muestran menores sentimientos de fracaso y tristeza (Oliver y Toner, 1990). En definitiva, esta perspectiva sugiere que la identificación con características instrumentales podría ser también ventajosa en el desarrollo de competencias de IE.

En línea con esta idea, diversas investigaciones han mostrado que la adscripción a un tipo de identidad de género estereotipada constituye una limitación para el desarrollo de una personalidad más adaptativa e implica un peor ajuste psicológico y bienestar subjetivo que una identidad más andrógina (Osofsky, Osofsky y Howard, 1972; Helgeson, 1994; Williams y D'Alessandro, 1994). El término androginia (Bem, 1974) supone una divergencia en la persona de los rasgos y características que se consideran apropiados para cada sexo. Además, implica que las cualidades que caracterizan a lo masculino (representado principalmente por rasgos de instrumentalidad) y a lo femenino (representado principalmente por rasgos de expresividad) no son opuestas, sino más bien complementarias. Por ello, tanto hombres como mujeres pueden incorporar elementos tanto "masculinos" como "femeninos" en su identidad (Parsons y Bales, 1955).

En base a estos planteamientos, algunos estudios han extendido el valor de la androginia al funcionamiento emocional y han planteado que el desarrollo de la IE podría ser una consecuencia de la capacidad de las personas de incorporar tanto rasgos estereotípicamente femeninos como rasgos estereotípicamente masculinos (Guastello y Guastello, 2003). Concretamente, se ha sugerido que las personas que se identifican tanto con rasgos de identidad expresivos como con rasgos de identidad instrumentales (es decir, las personas andróginas) muestran mayores niveles de IE que las personas con un tipo de identidad más estereotipada (Gartzia, 2010). Dado que esta relación positiva entre la androginia y la IE se ha establecido de manera preliminar y únicamente en relación a medidas de autopercepción, examinaremos si dicha relación se replica en el presente trabajo mediante el uso de diferentes medidas de IE.

Hipótesis 3: Las personas con un tipo de identidad de género andrógina (no estereotipada) mostrarán mayores niveles de IE que las personas con identidades estereotipadas.

\section{Método}

\section{Participantes}

En el estudio participaron 338 trabajadoras/es pertenecientes a veinte empresas, diez de ellas del sector industrial y diez del sector servicios. Su participación formaba parte de un programa de investigación-acción en competencias socioemocionales e innovación. En la primera sesión de dicho programa, las/os participantes debían responder a una serie de cuestionarios que incluían la medición de la identidad de género y la IE y, posteriormente, participaban en una serie de dinámicas sobre creatividad e innovación. La muestra incluía un $69.2 \%$ de hombres. La media de edad de las/os participantes fue de 38.34 años $(D T=9.13)$, con un rango de edades de 19 a 64, y presentaban la siguiente distribución en función del nivel de estudios: un $45.6 \%$ eran personas licenciadas, un $19.3 \%$ diplomadas y un $21 \%$ poseían un grado superior de FP. El 53 \% de las/os participantes ocupaba algún puesto de supervisión en la empresa.

\section{Instrumentos}

Bem Sex Role Inventory (BSRI; Bem, 1974): evalúa la identidad de género a través del grado de identificación con rasgos expresivos e instrumentales. En este estudio se utilizó una versión del instrumento adaptada al castellano (Echebarría y Pinedo, 1997). En dicha versión, se incluían 7 ítems para la escala de expresividad ( $\alpha$ de Cronbach=.77) y 13 ítems para la escala de instrumentalidad ( $\alpha$ de Cronbach=.74). Dada la creciente consideración de los ítems "masculina/o" y "femenina/o" como atributos independientes a los anteriores (Fernández, Quiroga, Del Olmo y Rodríguez, 2007), éstos fueron excluidos del análisis.

Personal Attributes Questionnaire (PAQ; Spence y Helmreich, 1978): es, después del BSRI, el instrumento más utilizado para la medición de la identidad de género y el que presenta mejores propiedades psicométricas (Beere, 1990). Está compuesto por 24 ítems en los que se debe indicar el grado de identificación con rasgos instrumentales, expresivos e instrumentalidad-expresividad. En el presente trabajo se utilizaron los factores de expresividad ( $\alpha$ de Cronbach $=.82)$ e instrumentalidad ( $\alpha$ de Cronbach $=.85$ ), cada uno de ellos compuesto por 8 ítems.

Tomando en consideración, por una parte, la coherencia interna observada entre el PAQ y el BSRI en la evaluación de rasgos expresivos e instrumentales en una revisión de 10 estudios en los que se utilizaron el BSRI y el PAQ de forma conjunta (Lenney, 1991), y por otra, las limitaciones que presentan ambos instrumentos para medir la identidad de género de forma aislada (Fernández et al., 2007), se unificaron los ítems de ambos instrumentos y se realizó un análisis de componentes principales sobre el conjunto de los ítems. La rotación Varimax arrojó un modelo de dos factores y los pesos factoriales resultantes fueron significativamente distintos de cero $(p \leq .01)$, dejando claro que los elementos de cada 
dimensión eran relevantes para la definición de su constructo correspondiente. El porcentaje de varianza explicada fue del 55\%. Las dimensiones de expresividad ( $\alpha$ de Cronbach $=.87 ; 7$ ítems del BSRI y 10 del PAQ) y de instrumentalidad ( $\alpha$ de Cronbach $=.84 ; 12$ ítems del BSRI y 5 del PAQ) resultantes de dicho análisis se tomaron como referencia. En tales dimensiones se incluyeron aquellos ítems con un peso factorial superior a .35 .

En relación a la IE, se utilizó el TMMS como medida de autopercepción y el MSCEIT como medida de habilidad.

Trait Meta Mood Scale (TMMS; Salovey, Mayer, Goldman, Turvey y Palfai, 1995): evalúa la IE autoinformada mediante las dimensiones de "atención emocional", representada por la capacidad de sentir y expresar los sentimientos de forma adecuada, "claridad emocional", caracterizada por una adecuada comprensión de los estados emocionales, y "reparación emocional", que evalúa la capacidad para regular los estados emocionales negativos sustituyéndolos por estados emocionales positivos. Para el presente estudio, se utilizó una versión reducida y adaptada al castellano del cuestionario que incluye los cuatro ítems de cada uno de los factores de la TMMS-24 (12 ítems en total) que muestran un mayor peso factorial, siempre superior a .40 (Salguero et al., 2009). Su estructura factorial conserva las dimensiones originales y muestra índices de consistencia interna adecuados $(\alpha$ de Cronbach para atención $=.86$; claridad $=.85$ y reparación $=.90)$.

Con el fin de igualar el formato de respuesta y reducir la incertidumbre asociada a los sujetos que responden con indecisión (Cheung y Mooi, 1994; González-Roma y Espejo, 2003), se utilizó una escala tipo Likert con un rango entre 1 y 6 en todos los instrumentos descritos.

Mayer-Salovey-Caruso Emotional Intelligence Test (MSCEIT; Mayer, Salovey y Caruso, 2000): es un instrumento de medida de la IE basado en las ramas de IE de percepción, facilitación, comprensión y regulación. Para la evaluación de esta habilidad, el cuestionario incluye 8 subescalas, 2 subescalas por rama. Debido a que en el presente estudio se utilizó una muestra de trabajadoras/es que respondió a los cuestionarios durante su jornada laboral, el tiempo con el que contaban para sus respuestas era limitado. Por este motivo, se decidió utilizar únicamente la rama de manejo de las emociones de la versión española del cuestionario (Extremera, Fernández-Berrocal, y Salovey, 2006). Esta dimensión presenta índices de fiabilidad adecuados (.83 para la fiabilidad consenso y .81 para la fiabilidad experta/o) e incluye las subescalas de "manejo de las emociones propias" y de "manejo de las emociones ajenas".

\section{Resultados}

\section{Diferencias sexuales en identidad de género}

En primer lugar, se llevó a cabo un análisis de comparación de medias entre las puntuaciones obtenidas por las mujeres y los hombres en las dimensiones de expresividad e ins- trumentalidad de la identidad de género, así como en todas las variables de IE estudiadas. Como se muestra en la tabla 1 , se observan diferencias estadísticamente significativas y de magnitud moderada en las dimensiones de expresividad, manejo de las emociones propias, y manejo de las emociones ajenas. En todos los casos, las mujeres muestran puntuaciones superiores a los hombres. Estos datos confirman, por lo tanto, la hipótesis 1, que establecía que las mujeres se identificarían en mayor medida que los hombres con rasgos de identidad expresivos asociados a las competencias de IE (véanse las correlaciones entre todas las variables analizadas en la tabla 2). También se observó, en relación a la variable edad, una correlación estadísticamente significativa con la dimensión de regulación de las emociones ajenas, observándose que, a mayor edad, menor habilidad para ayudar a otras personas a manejar sus estados emocionales.

Tabla 1. Comparaciones entre las medias obtenidas por las mujeres y por los hombres en las variables que configuran la IE y la identidad de género.

\begin{tabular}{|c|c|c|c|c|c|c|}
\hline \multirow[t]{2}{*}{ Variables } & \multicolumn{2}{|c|}{ Mujeres } & \multicolumn{2}{|c|}{ Hombres } & \multirow[t]{2}{*}{$t$} & \multirow[t]{2}{*}{$d$} \\
\hline & Media & $D T$ & Media & $D T$ & & \\
\hline Atención Emocional & 3.81 & 0.74 & 3.77 & 0.82 & 0.39 & 0.07 \\
\hline Claridad Emocional & 4.20 & 0.64 & 4.04 & 0.70 & 1.86 & 0.24 \\
\hline Reparación Emocional & 4.03 & 0.78 & 4.14 & 0.78 & -1.18 & 0.14 \\
\hline $\begin{array}{l}\text { Regulación Emociones } \\
\text { Propias }\end{array}$ & 93.98 & 9.74 & 89.07 & 9.08 & $4.09 * *$ & 0.52 \\
\hline $\begin{array}{l}\text { Regulación Emociones } \\
\text { Ajenas }\end{array}$ & 94.85 & 11.45 & 89.79 & 11.01 & $3.51 * *$ & 0.45 \\
\hline Expresividad & 4.68 & 0.54 & 4.43 & 0.51 & $4.01 * *$ & 0.48 \\
\hline Instrumentalidad & 4.21 & 0.51 & 4.17 & 0.50 & 0.81 & 0.11 \\
\hline
\end{tabular}

\section{Papel mediador de la expresividad}

Con el fin de analizar la segunda de las hipótesis, que establecía que la aceptación de rasgos de expresividad podría mediar la relación entre el sexo y la IE, se llevaron a cabo una serie de análisis de regresión siguiendo el modelo de Baron y Kenny (1986) en combinación con pruebas Sobel ${ }^{2}$. Dado que no se encontraron diferencias estadísticamente significativas entre mujeres y hombres en las dimensiones de IE medidas con el TMMS, los análisis de mediación se limitaron a las dimensiones de IE medidas con el MSCEIT. En primer lugar, se examinó el posible efecto mediador de la expresividad sobre la regulación de las emociones propias. El primer paso consistía en comprobar el efecto de la variable predictora (sexo) sobre la variable criterio (regulación de las emociones propias). Este paso se confirmó al incluir ambas variables en la ecuación de regresión $(\beta=-4.90$; ES $=1.20, p \leq .01)$. En segundo lugar, se encontró un efecto principal del sexo sobre la expresividad $(\beta=-0.25, E S=0.06$,

\footnotetext{
2 A pesar de que nuestra hipótesis de partida se basaba en análisis de mediación, y no de moderación, se llevaron a cabo análisis de la varianza para descartar el efecto moderador de la expresividad sobre las dimensiones de IE. En dichos resultados de moderación, no se observó ningún efecto de interacción estadísticamente significativo entre las variables predictoras (es decir, el sexo y la expresividad).
} 
$p \leq .01$ ), confirmando así el segundo paso del modelo de mediación. Por último, los resultados mostraron que la expresividad permite predecir la regulación de las emociones propias aún habiendo controlado el efecto del sexo en la ecuación de regresión $(\beta=2.83, E S=1.07, p \leq .01)$, confirmando así el último paso en el modelo de mediación (Baron y Kenny, 1986). Si bien la prueba de Sobel para la disminución de la $\beta$ fue estadísticamente significativa, $z=2.23, p \leq .05$ para una prueba unilateral, el efecto del sexo en esta dimensión de la IE siguió siendo estadísticamente significativo tras incluir la dimensión de expresividad en el modelo $(\beta=-4.20$, $E S=1.21, p \leq .01)$, lo que indica que la mediación es parcial.

Tabla 2. Correlaciones entre las variables analizadas.

\begin{tabular}{|c|c|c|c|c|c|c|c|}
\hline & 1 & 2 & 3 & 4 & 5 & 6 & 7 \\
\hline 1. Atención Emocional & - & & & & & & \\
\hline 2. Claridad Emocional & $.47^{* *}$ & - & & & & & \\
\hline $\begin{array}{l}\text { 3. Reparación Emocio- } \\
\text { nal }\end{array}$ & $.31^{* *}$ & $.41^{* *}$ & - & & & & \\
\hline $\begin{array}{l}\text { 4. Regulación Emocio- } \\
\text { nes Propias }\end{array}$ & .06 & $.17^{* *}$ & $.15^{*}$ & - & & & \\
\hline $\begin{array}{l}\text { 5. Regulación Emocio- } \\
\text { nes Ajenas }\end{array}$ & .06 & $.16^{* *}$ & $.13^{*}$ & $.60^{* *}$ & - & & \\
\hline 6. Expresividad & $.28^{* *}$ & $.27^{* *}$ & $.28^{* *}$ & $.20^{* *}$ & $.19^{* *}$ & - & \\
\hline 7. Instrumentalidad & $.20^{* *}$ & $.23^{* *}$ & $.23^{* *}$ & -.03 & -.01 & $.22^{* *}$ & - \\
\hline 8. Edad & -.01 & -.07 & .04 & -.08 & $-.14^{*}$ & -.11 & .05 \\
\hline
\end{tabular}

Para examinar si la expresividad mediaba la relación entre el sexo y la regulación de las emociones ajenas, se llevaron a cabo nuevos análisis de regresión (Baron y Kenny, 1986). El primer paso consistía en analizar el efecto del sexo sobre la regulación de las emociones ajenas. Este efecto fue estadísticamente significativo, confirmando así el segundo paso del modelo de mediación $(\beta=-5.06 ; \mathrm{ES}=1.43, p \leq .01)$. Habiendo confirmado previamente el segundo paso del modelo (es decir, el efecto del sexo sobre la expresividad), se procedió a introducir la variable mediadora de expresividad en el modelo. Los resultados mostraron que la expresividad predecía la regulación de las emociones ajenas, incluso cuando se controlaba el efecto del sexo $(\beta=3.20$, ES $=1.29$, $p \leq .05)$. La prueba de Sobel fue significativa, $z=2.13, p \leq .05$ para una prueba unilateral, confirmando así el papel mediador de los rasgos expresivos de identidad en la regulación de las emociones ajenas. Sin embargo, el efecto del sexo se mantuvo estadísticamente significativo $(\beta=-4.26$, ES $=$ $1.46, p \leq .01)$, lo que sugiere que el efecto de mediación previsto en este caso también es parcial.

\section{Androginia e Inteligencia emocional}

Con objeto de comprobar la tercera de las hipótesis planteadas, que establecía que las personas con mayor aceptación de características de identidad de ambos tipos (andróginas) presentarían niveles superiores de IE que aquellas personas con un tipo de identidad de género más estereotipada, se llevaron a cabo diversos análisis de covarianza. Para ello, se creó una variable categórica con tres dimensiones: identidad de género expresiva (asignada a los individuos con puntuaciones por encima de la media en expresividad y por debajo de la media en instrumentalidad), instrumental (asignada a los individuos con puntuaciones por encima de la media en instrumentalidad y por debajo de la media en expresividad) y andrógina (asignada a los individuos con puntuaciones por encima de la media en ambas dimensiones). En primer lugar, se tomó la identidad de género como variable predictora y la atención, claridad y reparación, respectivamente, como variables criterio. Asimismo, se controló el efecto del sexo y la edad en dichos análisis.

En relación a la atención emocional, los resultados mostraron que la identidad de género ejerce una influencia estadísticamente significativa sobre dicha dimensión, $\mathrm{F}(3$, $317)=9.57$; $p \leq .01)$. Las comparaciones múltiples a posteriori llevadas a cabo mediante la prueba de Tukey pusieron de manifiesto que existen diferencias estadísticamente significativas $(p \leq .01)$ y de magnitud moderada ( $g$ de Hedges $=.56$ ) entre las personas andróginas $(M=4.10, S D=0.72)$ y las personas instrumentales $(M=3.67, S D=0.74)$, siendo los sujetos andróginos los que puntuaron más alto. Por otra parte, aunque no se observaron diferencias estadísticamente significativas entre los individuos expresivos y los instrumentales, el tamaño del efecto asociado a la diferencia de medias adoptó un valor de .35 , siendo los expresivos los que puntuaron más alto.

Para la claridad emocional, los resultados del ANCOVA también mostraron un efecto estadísticamente significativo del tipo de identidad de género, $\mathrm{F}(3,319)=12.49 ; p \leq .01$, observándose diferencias estadísticamente significativas $(p \leq .01)$ y de magnitud moderada ( $g$ de Hedges $=.70$ ) entre las personas andróginas $(M=4.44, S D=0.63)$ y las instrumentales $(M=3.98, S D=0.63)$. De forma similar, se observaron diferencias estadísticamente significativas $(p \leq .05)$ y de magnitud moderada ( $g$ de Hedges $=.53$ ) entre las personas andróginas y las expresivas $(M=4.10, S D=0.64)$. En ambos casos fueron los sujetos andróginos los que obtuvieron puntuaciones mayores.

En el caso de la reparación emocional, los resultados también mostraron una influencia estadísticamente significativa del tipo de identidad de género, $\mathrm{F}(3,322)=11.65 ; p \leq .01$, observándose, al igual que en el caso anterior, diferencias estadísticamente significativas entre los sujetos andróginos $(M$ $=4.50, S D=0.79)$ y los expresivos $(M=4.02, S D=0.66) \mathrm{y}$ entre los andróginos e instrumentales $(M=3.95, S D=0.74)$ $(p \leq .01$ en ambos casos). Los tamaños del efecto asociados a tales diferencias de medias fueron de magnitud moderada en los dos casos ( $g$ de Hedges $=0.64$ y 0.73 , respectivamente), siendo las personas andróginas las que obtuvieron puntuaciones mayores.

Con el fin de comprobar si la identidad de género andrógina también se asociaba a niveles mayores de IE en el caso de la regulación emocional medida con el MSCEIT, se realizaron nuevos análisis de la covarianza, en los que se tomó la identidad de género como variable predictora y la regulación 
de las emociones propias y la regulación de las emociones ajenas como variables criterio. En este caso, también se controló el efecto del sexo y la edad. Los resultados mostraron que la identidad de género ejerce un efecto estadísticamente significativo tanto sobre la habilidad para manejar las emociones propias $(\mathrm{F}(3,272)=3.77, p \leq .05)$ como sobre la habilidad para manejar las emociones ajenas $(\mathrm{F}(3,272)=3.62$, $p \leq .05)$.

De nuevo, las comparaciones múltiples a posteriori pusieron de manifiesto que los individuos andróginos mostraban puntuaciones más altas que los instrumentales en ambos casos $(p \leq .05$ en la prueba de Tukey y $g$ de Hedges $=.47$ ) (ver figura 1). En relación a los individuos expresivos, se encontró una tendencia específica. Respecto a la rama de regulación de las emociones ajenas, no se encontraron diferencias entre los individuos expresivos $(M=93.27, S D=9.03)$ y los andróginos $(M=94.31, S D=12.47)$ ( $g$ de Hedges $=$. 05). Sin embargo, los individuos expresivos mostraron puntuaciones superiores a las de los instrumentales $(M=89.01$, $S D=11.30)$ en esta habilidad ( $p \leq .05$ en la prueba de Tukey; $g$ de Hedges $=.47)$. Esta tendencia no aparece en el caso del manejo de las emociones propias, donde la diferencia entre los individuos andróginos $(M=93.26, S D=10.31)$ y los expresivos $(M=90.65, S D=9.13)$ es algo mayor que en el caso de la gestión de las emociones ajenas $(g$ de Hedges $=.28$ ) y la diferencia entre los individuos expresivos e instrumentales $(M=88.45, S D=9.44)$ no es tan marcada $(p>.05 ; g$ de Hedges $=.22)$.

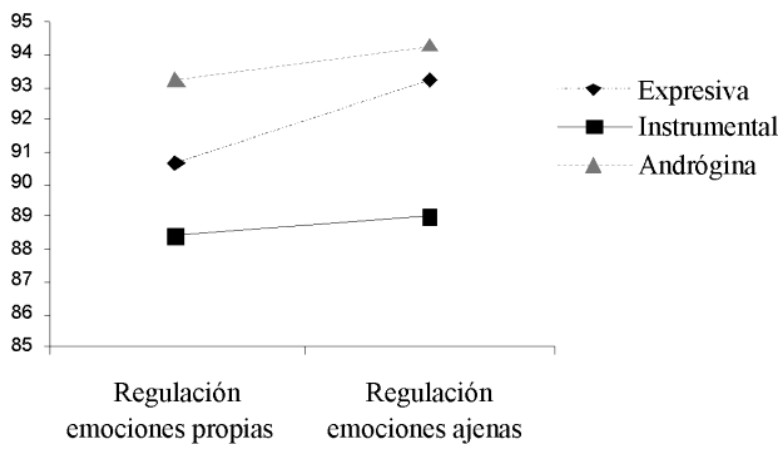

Figura 1. Niveles de inteligencia emocional (medidas con el MSCEIT) en función del tipo de identidad de género.

Con objeto de examinar de manera más exhaustiva si la aceptación de rasgos de identidad contra-estereotípicos se relaciona positivamente con la IE, se creó una nueva variable con sólo dos dimensiones: identidad estereotipada, que fue asignada a los hombres instrumentales (aquellos que puntuaban por encima de la media en instrumentalidad y por debajo de la media en expresividad) y a las mujeres expresivas (aquellas que puntuaban por encima de la media en expresividad y por debajo de la media en instrumentalidad), e identidad no estereotipada, que se asignó a los hombres expresivos o andróginos y a las mujeres instrumentales o andróginas. Los resultados de la comparación entre las medias llevada a cabo mediante la $t$ de student mostraron diferencias estadísticamente significativas y de magnitud moderada entre la identidad estereotipada y la no estereotipada en todas las dimensiones de la IE en el caso del TMMS. En cuanto al MSCEIT, también se observaron tales diferencias, aunque los tamaños del efecto fueron algo menores (ver tabla 3). En todos los casos, los sujetos con identidades no estereotipadas presentaron niveles superiores de IE que los sujetos con identidades estereotipadas. Estos datos confirman la hipótesis 3.

Tabla 3. Comparaciones entre las medias de los sujetos con identidad estereotipada e identidad no estereotipada en las dimensiones de IE.

\begin{tabular}{|c|c|c|c|c|c|c|}
\hline \multirow[t]{2}{*}{ TMMS } & \multicolumn{2}{|c|}{$\begin{array}{c}\text { Identidad } \\
\text { estereotipada } \\
\end{array}$} & \multicolumn{2}{|c|}{$\begin{array}{l}\text { Identidad no } \\
\text { estereotipada }\end{array}$} & \multirow[b]{2}{*}{$t$} & \multirow[b]{2}{*}{$d$} \\
\hline & Media & $D T$ & Media & $D T$ & & \\
\hline Atención & 3.66 & 0.76 & 4.00 & 0.731 & $-3.55 * *$ & 0.46 \\
\hline Claridad & 3.97 & 0.65 & 4.31 & 0.63 & $-4.05 * *$ & 0.53 \\
\hline Reparación & 3.93 & 0.74 & 4.32 & 0.77 & $-3.96 * *$ & 0.52 \\
\hline \multirow[t]{2}{*}{ MSCEIT } & \multicolumn{2}{|c|}{$\begin{array}{c}\text { Identidad } \\
\text { estereotipada } \\
\end{array}$} & \multicolumn{2}{|c|}{$\begin{array}{l}\text { Identidad no } \\
\text { estereotipada }\end{array}$} & & \\
\hline & Media & $D T$ & Media & $D T$ & $t$ & $d$ \\
\hline $\begin{array}{l}\text { Regulación Emo- } \\
\text { ciones Propias }\end{array}$ & 88.73 & 8.98 & 91.87 & 10.29 & $-2.29 *$ & 0.33 \\
\hline $\begin{array}{l}\text { Regulación Emo- } \\
\text { ciones Ajenas }\end{array}$ & 89.65 & 10.23 & 93.10 & 12.30 & $-2.14^{*}$ & 0.31 \\
\hline
\end{tabular}

\section{Discusión}

Un gran número de estudios ha señalado que existen diferencias sexuales en el desarrollo de la Inteligencia Emocional (IE). En particular, la mayoría de investigaciones que han estudiado este tipo de inteligencia analizando las diferencias entre mujeres y hombres han mostrado que las mujeres tienden a presentar mayores niveles de IE, especialmente cuando se utilizan medidas de habilidad como el MSCEIT (Mayer, Salovey y Caruso, 2000; Salovey y Mayer, 1990; Salovey, 2006). Los resultados del presente trabajo sugieren que dichas diferencias se deben, al menos en parte, a la mayor identificación de las mujeres con rasgos de identidad expresivos. Así, hemos mostrado que las mujeres tienden a identificarse en mayor medida que los hombres con rasgos expresivos de identidad asociados a competencias de IE y que dicha identificación media parcialmente la relación entre el sexo y la IE -las mujeres podrían ser emocionalmente más inteligentes debido a que se identifican en mayor medida con rasgos de expresividad. Sin embargo, en nuestro estudio, el papel mediador de la expresividad sólo se confirma en el caso de la IE medida a partir de cuestionarios de habilidad y se ha limitado al estudio de la regulación emocional. Por lo tanto, sería necesario plantear nuevos estudios que analicen el potencial papel mediador de la expresividad utilizando diferentes muestras y distintas medidas de evaluación de la IE. Asimismo, la relación negativa encontrada entre la edad y la regulación de las emociones ajenas debería ser contrastada en futuras investigaciones, ya que estudios previos han mostrado que, al menos en el caso de la regulación de las emo- 
ciones propias, las personas de mayor edad tienden a desarrollar mayores habilidades de regulación emocional (Márquez-González et al., 2008).

En el caso de la IE evaluada a partir de medidas de autopercepción, no se ha podido evaluar el papel mediador de la expresividad ya que no se han observado diferencias estadísticamente significativas entre las mujeres y los hombres en las dimensiones de IE. Estos resultados pueden deberse a que, tal y como se ha apuntado en otros estudios (Wilson y Dunn 2004), las medidas de IE basadas en la autopercepción no se corresponden, de manera tan directa como las medidas de habilidad, con las conductas observadas en situaciones reales, siendo más consistentes las diferencias sexuales encontradas con medidas de habilidad tales como el MSCEIT (Extremera, Fernández-Berrocal, y Salovey, 2006; Salguero et al., 2009; Salovey, 2006). En cualquier caso, los resultados del presente trabajo confirman que, en línea con lo previsto, la identidad de género ejerce influencia sobre la IE evaluada tanto a través de medidas de autopercepción (TMMS) como de habilidad (MSCEIT). Concretamente, los resultados corroboran que, a diferencia de otros tipos de identidad de género, la identidad andrógina se relaciona con niveles superiores de IE en todas las dimensiones analizadas. Estos datos coinciden con los obtenidos en otros estudios (Guastello y Guastello, 2003) y sugieren que la identidad de género puede ser un mejor predictor de las diferencias sexuales en IE que el sexo. Sin embargo, cabe señalar que nuestros resultados relativos a la IE evaluada a partir de medidas de habilidad se han limitado a la dimensión de manejo de las emociones. Por lo tanto, sería conveniente que futuros estudios examinen el papel de la identidad de género en relación al resto de dimensiones de IE.

Los resultados expuestos abren importantes interrogantes para la investigación sobre el género y las emociones. Por ejemplo, cabría preguntarse si la identidad de género y los roles asociados a ella determinan, en mayor medida que el sexo, las diferencias entre mujeres y hombres en IE o si es posible favorecer el desarrollo de las competencias emocionales de mujeres y hombres a través de la incorporación de rasgos de identidad de género contra-estereotípicos. De hecho, el patrón de resultados encontrado en este trabajo sugiere que las mujeres y los hombres que muestran un tipo de identidad menos estereotipada muestran niveles superiores de IE que las personas con un tipo de identidad de género más acorde a su sexo. Estos resultados son coherentes con estudios previos que ponen de manifiesto la importancia de la identidad de género andrógina para una mayor autoestima y ajuste psicológico (Osofsky, Osofsky y Howard, 1972; Williams y D'Alessandro, 1994). A pesar de ello, y dado que se ha utilizado un tipo de diseño transversal, no se puede establecer de manera inequívoca la dirección de las relaciones encontradas, pudiendo darse la posibilidad de que, por ejemplo, la IE mediara la relación existente entre el sexo y la identidad de género.

Dado que el estudio de las competencias emocionales en función del género, al igual que el de otras variables psicoló- gicas como la personalidad, las habilidades de liderazgo, la autoestima, la agresividad o el razonamiento moral, se basa, habitualmente, en establecer diferencias entre mujeres y hombres desde un "enfoque de la diferencia sexual" (Hyde, 2005; Stewart, y McDermott, 2004; Rocha-Sánchez y DíazLoving, 2005), consideramos que los resultados de este trabajo pueden ser de gran relevancia en el ámbito de estudio del género y la IE. Así, mientras que los estudios realizados hasta ahora se han orientado a examinar las diferencias sexuales en IE, en el presente trabajo se muestra la necesidad de atender a factores contextuales relacionados con los roles de género y la identidad. Por ello, parece pertinente que se propongan nuevas definiciones de la identidad de mujeres y hombres, así como de la forma en la que ambos vivencian y expresan sus emociones, ya que dicha flexibilización de los roles de género podría facilitar el despliegue de respuestas emocionales más adaptativas y menos determinadas por el sexo de las personas (Gartzia, 2012).

Por otra parte, en base a los resultados observados, parece necesario reforzar rasgos de identidad contraestereotípicos en el caso de los hombres. Los datos muestran que, al contrario de lo que ocurre con las variables de instrumentalidad, existen importantes diferencias entre mujeres y hombres en la adscripción a rasgos de expresividad, siendo esta adscripción menor en el caso de los hombres. Este patrón es coherente con resultados encontrados previamente (López-Zafra, García-Retamero, Diekman y Eagly, 2008; Twenge, 1997). Dado que hemos mostrado que la aceptación de rasgos de expresividad podría ser clave para un mayor desarrollo de la IE, parece pertinente que se continúe trabajando, a nivel aplicado, en la deconstrucción del modelo de masculinidad exclusivamente instrumental reforzado clásicamente en nuestra sociedad. Este trabajo podría llevarse a cabo a través de campañas de corresponsabilidad o de la progresiva implicación de los hombres en las acciones orientadas a favorecer la igualdad de oportunidades de hombres y mujeres.

Por último, es importante recordar que las organizaciones laborales, en las que se contextualiza este estudio, constituyen ámbitos masculinizados, lo cual conlleva que las personas que en ellas se desenvuelven adopten rasgos y conductas estereotípicamente masculinas o instrumentales (Gartzia, 2010; Gartzia, Ryan, Balluerka y Aritzeta, 2011; Munduate, 2003). Dado que las competencias socioemocionales son una herramienta esencial en las organizaciones (Brief y Weiss, 2002; García-Izquierdo, García-Izquierdo y RamosVillagrasa, 2007; Piñar-Chelso y Fernández-Castro, 2011), creemos que resulta necesario promover un modelo organizacional menos caracterizado por la instrumentalidad y los valores estereotípicamente masculinos ya que se contraponen, por su propia definición, al desarrollo de muchas de dichas competencias (Gartzia, 2011; Gartzia y van Knippenberg, 2011; Eagly, Gartzia y Carli, 2012). Así, en la medida en que vayan desapareciendo los prejuicios sociales contra las mujeres, se irá promoviendo una mayor aceptación de rasgos estereotípicamente femeninos y produciendo el cam- 
bio que el contexto socioeconómico actual precisa. En definitiva, consideramos que esta inclusión de la perspectiva de género en el estudio de las competencias socioemocionales y la IE resulta clave para favorecer referentes individuales y grupales más acordes a los requerimientos de la sociedad actual.

\section{Referencias}

Baron, R. M., y Kenny, D. A. (1986). The moderator-mediator variable distinction in social psychological research: Conceptual, strategic, and statistical considerations. Journal of Personality and Social Psychology, 6, 11711182.

Bar-On, R. (2006). The Bar-On model of emotional-social intelligence (ESI). Psicothema, 18, 13-25.

Beere, C.A. (1990). Gender roles: A Handbook of tests and measures. Westport, CT: Greenwood.

Bem, S.L. (1974). The measurement of psychological androgyny. Journal of Consulting and Clinical Psychology, 42, 155-62.

Blazina, C. (2001). Analytic Psychology and gender role conflict: The Development of the fragile masculine self. Psychotherapy, 38, 50-59.

Bourne, V. J., y Maxwell, A. M. (2010). Examining the sex difference in lateralisation for processing facial emotion: Does biological sex or psychological gender identity matter?. Neuropsychologia 48, 1289-1294

Bracket, M.A., y Salovey, P. (2006). Measuring emotional intelligence with the Mayer-Salovey-Caruso emotional intelligence test (MSCEIT). Psycothema, 18, 34-41.

Brief, A.P., y Weiss, H.M. (2002). Organizational behaviour: Affect in the workplace. Annual Review of Psychology, 53(1), 279-307.

Brody, L.R., y Hall, J.A. (2000). Gender, emotion, and expression. En: M. Lewis y J.M. Haviland-Jones (Ed.), Handbook of emotions (pp.338-349). New York: Guilford Press.

Candelá, C., Barberá, E., Ramos, A., y Sarrió, M. (2002). Inteligencia Emocional y la variable género, Revista Electrónica de Motivación y Emoción, 5, 10.

Cheung, K.C., y Mooi, L.C. (1994). A comparison between the rating scale model and dual scaling for Liker $t$ scales. Applied Psychological Measurement, 18, 1-13.

Conway, M. (2000). On sex roles and representation of emotional experience: masculinity, femininity and emotional awareness. Sex Roles, 43(910), 687-698.

Conway, M., Giannopoulos, C., y Stiefenhofer, K. (1990). Response styles to sadness are related to sex and sex-role orientation. Sex Roles, 22, 579587.

Dambrun, M., Duarte, S., y Guimond, S. (2004). Why are men more likely to support group-based dominance that women? The mediating role of gender identification. British Journal of Social Psychology, 43, 287-297.

Dawda, D., y Hart, S. (2000). Assessing emotional intelligence: reliability and validity of the Bar-On Emotional Quotient Inventory (EQ-I) in university students. Personality and Individual Differences, 28(4), 797-812.

Eagly, A. H. (1987). Sex differences in social behavior: a social interpretation. Hillsdale, NJ: LEA.

Eagly. A. H., y Karau, S. J. (2002) Role congruity theory of prejudice toward female leaders. Psychological Review, 109, 573-598.

Eagly, A. H., Gartzia, L., y Carli, L. (2012). Female advantage: revisited. En S. Kumra, R. Simpson y R. Burke (Ed.), The Oxford Handbook of Gender in Organizations. Oxford University Press.

Echebarria, A. (2010). Role Identities versus Social Identities: Masculinity, Femininity, Instrumentality and Communality. Asian Journal of Social Psychology, 13(1), 30-43.

Echebarría, A., y Pinedo, J.A. (1997). Identidad social de género: Su distribución social e influencia en el juicio. Revista de Psicología Social, 12, 131151.

Extremera, N., Fernández-Berrocal, P., y Salovey, P. (2006). Spanish version of the Mayer-Salovey-Caruso Emotional Intelligence Test (MSCEIT) version 2.0: reliabilities, age and gender differences. Psicothema, 18, supl., $42-48$.
Agradecimientos.- Este estudio ha sido posible gracias a la financiación del Departamento de Educación, Universidades e Investigación del Gobierno Vasco, a través de la concesión de una beca predoctoral para la realización de la tesis doctoral de Leire Gartzia, y a la Diputación Foral de Guipúzcoa (Departamento de Innovación y Sociedad del Conocimiento, OF94/2008).

Fernández, J., Quiroga, M.A., Del Olmo, I., y Rodríguez, A. (2007). Escalas de masculinidad y feminidad: estado actual de la cuestión. Psicothema, 19(3), 357-365.

Fischer, A.H. (1993). Sex differences in emotionality: Fact or stereotype? Feminism and Psychology, 3, 303-318.

Friedman, H.S., y Riggio, R.E. (1999). Individual differences in the ability to encode complex affects. Personality and Individual Differences, 27, 182-194.

García-Izquierdo, L., García-Izquierdo, M. y Ramos-Villagrasa, P.J. (2007). Aportaciones de la inteligencia emocional y la autoeficacia: Aplicaciones para la selección de personal. Anales de Psicología, 23(2), 231-239.

Gartzia, L. (2010). From "Thik Male" to "Think Androgynous". European Dissertation, University of the Basque Country. UPV/EHU Editorial Service.

Gartzia, L., Ryan, M.K., Balluerka, N., y Aritzeta, A. (2011). Think crisisthink female: further evidence. European Journal of Work and Organizational Psychology, Oct., 1-26.

Gartzia, L. y van Knippenberg, D. (2011). The Gendered nature of (male) leadership: Expressive identity salience and cooperation. Best Paper Proceedings of the Academy of Management Annual Meeting, San Antonio, Texas.

Gartzia, L. (2011). Nuevos modelos de gestión: de pensar en masculino a pensar en andrógino. Boletín de Estudios Económicos, 203, 299-314.

Gartzia, L. (2012). La maleabilidad del género: efectos de la exposición breve a imágenes contra-estereotípicas en materia afectivo-sexual. Revista de Psicología Social. En prensa.

González-Roma, V., y Espejo, B. (2003). Análisis de las categorías de respuesta central "No estoy seguro", "término medio" y "?" en ítems politómicos. Psicothema, 15(2), 278-284.

Guastello, D.D., y Guastello, S.J. (2003). Androgyny, Gender Role Behavior and Emotional Intelligence among collage students and their parents. Sex Roles, 49(11/12), 663-673.

Helgeson, V. S. (1994). Relation of agency and communion to well-being: Evidence and potential explanations. Psychological Bulletin, 116, 412-428.

Hoffman, M.L. (1977). Sex differences in empathy and related behaviours. Psychological Bulletin, 84, 712-722.

Hyde, J.S. (2005). The gender similarities Hypothesis. American Psychologist, 60(6), 581-592.

Joseph, D.L., y Newman, D.A. (2010). Emotional Intelligence: An Integrative Meta-Analysis and Cascading Model. Journal of Applied Psychology, 95(1), 54-78

Lenney, E. (1991). Sex roles the measurement of masculinity, femininity, and androgyny. En J.P. Robinson, P.R. Shaver, y L.S. Wrightsman (Ed.), Measures of psychological attitudes (pp.573-660). San Diego: Academic Press.

López-Zafra, E., García-Retamero, R., Diekman, A., y Eagly, A.H. (2008). Dinámica de estereotipos de género y poder: un estudio transcultural. Revista de Psicología Social, 23(2), 213-219.

Mayer, J.D., Salovey, P., y Caruso, D.R. (2000). Mayer-Salovey-Caruso Emotional Intelligence Test (MSCEIT) item blocket, Research version. Toronto Ontario, Canada: MHS Publishers.

Márquez-González, M., Izal, M., Montorio, I. y Losada, A. (2008). Experiencia y regulación emocional a lo largo de la etapa adulta del ciclo vital: análisis comparativo en tres grupos de edad. Psicothema, 20, 616-622.

Munduate, L. (2003). Género y Liderazgo. Diferencias entre hombres y mujeres en el acceso a puestos directivos. Revista de Psicología Social, 18(3), 309-314.

Oliver, S. J., y Toner, B. B. (1990). The influence of gender role typing on the expression of depressive symptoms. Sex Roles, 22, 775-790. 
Osofsky, J.D., y Osofsky, H.J, y Howard (1972). Androgyny as a Life Style, Family Coordinator, 21, 411-418.

Parsons, T., y Bales, R. F. (1955). Family, socialization and interaction process. Nueva York: Free Press.

Petrides, K. V., Furnham, A., y Martin, G. N. (2004). Estimates of emotional and psychometric intelligence: Evidence for gender-based stereotypes. The Journal of Social Psychology, 144, 149-162.

Piñar-Chelso, M.J. y Fernández-Castro, J. (2011). La influencia de la inteligencia emocional en el estrés, la disonancia emocional y el rendimiento de tripulantes de cabina de pasajeros. Anales de Psicología, 27(1), 65-70.

Randel, A. E. (2002). Identity salience: A moderator of the relationship between group gender composition and work group conflict. Journal of Organizational Behavior, 23(6), 749-766.

Reevy, G. M. y Maslach, C. (2001). Use of social support: gender and personality differences. Sex Roles, 44(7/8), 437-459.

Rocha-Sánchez, T.E., y Díaz-Loving, R. (2005). Cultura de género: la brecha ideológica entre mujeres y hombres. Anales de Psicología, 21(1), 42-49.

Salguero, J.M., Fernández-Berrocal, P., Ruíz-Aranda, D., y Cabello, R. (2009). Propiedades psicométricas de la versión reducida de la Trait Meta-Mood Scale: TMMS-12. In Fernández-Berrocal, P., Extremera, N., Palomera, R., Ruiz-Aranda, D., Salguero, J.M., y Cabello, R. (Coord.). Avances en el estudio de la Inteligencia Emocional (pp. 129-133). Fundación Marcelino Botín.

Salovey, P., y Mayer, J.D. (1990). Emotional Intelligence. Imagination, Cognition, and Personality, 9, 185-211.

Salovey, P., Mayer, J.D., Goldman, S.L., Turvey, C., y Palfai, T.P. (1995) Emotional attention, clarity and repair: exploring emotional intelligence using the Trait Meta-Mood Scale. En J.W. Pennebaker (Ed.), Emotion, disclosure, and health (pp.125-154). Washington, DC: APA.

Salovey, P. (2006). Epilogue: The Agenda for Future Research. In V. Druskat; F. Sala; G. Mount (Ed.), Linking EI and Performance at Work -
Current Research Evidence with Individuals and Groups (pp. 267-272). LEA Inc.

Sánchez, M.T., Fernández-Berrocal, P., Montañés, R., y Latorre, J.M. (2008). ¿Es la inteligencia emocional una cuestión de género? Socialización de las competencias emocionales en hombres y mujeres y sus implicaciones. Revista Electrónica de Investigación Psicoeducativa, 15(2), 455-474.

Spence, J.T., y Helmreich, R.L. (1978). Masculinity and femininity: Their psychological dimensions, correlates and antecedents. Austin: University of Texas Press.

Stewart, J., y Mcdermott, C. (2004). Gender in psychology. Annual Review of Psychology, 55, 519-544.

Tajfel, H., y Turner, J.C. (1986). The social identity theory of inter-group behavior. En S. Worchel and L.W. Austin (eds.), Psychology of Intergroup Relations. Chigago: Nelson-Hall

Twenge, J.M. (1997). Changes in Masculine and Feminine Traits over time: A meta-Analysis. Sex Roles, 36(5-6), 305-325.

Twenge, J.M. (2001). Changes in women's assertiveness in response to status and roles: A cross-temporal meta-analysis, 1931-1993. Journal of Personality and Social Psychology, 81, 133-145.

Wester, S.R., Vogel, D.L., Pressly, P., y Heesacker, M. (2002). Sex differences in emotion: A critical Review of the literature and implications for counselling Psychology. The counselling Psychologist, 30(4), 630-652.

Williams, D.E., y D'alessandro, J.D. (1994). A comparison of three measures of androgyny and their relationship to psychological adjustment. Journal of Social Behavior and Personality, 9, 469-480.

Wilson, T.D., y Dunn, E. (2004). Self-knowledge: Its limits, value, and potential for improvement. Annual Review of Psychology, 55, 493-518.

(Artículo recibido: 29-03-2011, revisión: 22-10-2011, aceptado: 26-10-2011) 